\title{
TRENDS IN BIRD SPECIES RICHNESS IN THE MIDST OF DROUGHT
}

BRIAN M. MYERS, Department of Biological Sciences, San Diego State University, 5500 Campanile Drive, San Diego, California 92182; bmyers@mail.sdsu.edu

ERIN J. QUESTAD, MARCUS D. HUBBELL, and DAVID J. MORIARTY, Department of Biological Sciences, California State Polytechnic University, Pomona, 3801 W Temple Ave, Pomona, California 91768

ABSTRACT: Climate change is predicted to exacerbate the effects of disturbances such as drought on numerous wildlife communities. On the basis of surveys from 1981 to 2014 , we investigated whether drought altered the species richness and composition of bird communities of coastal sage scrub in two protected areas of southern California. At one site, the Voorhis Ecological Reserve, Pomona, we found that the number of species of permanent residents, but not of summer and winter visitors, was lower during droughts than during periods of at least average rainfall. At the other site, the Bernard Field Station, Claremont, we found that the richness of resident species remained the same in both drought and nondrought periods, and richness of summer and winter visitors increased during times of drought. The difference in patterns between these sites may be explained by the presence of a constructed, permanent water source at the second site. Thus, supplemental water sources embedded in natural areas might be an important resource for native bird species during drought.

Climate change is predicted to increase the frequency of drought in arid regions, threatening avian populations and native ecosystems (National Wildlife Federation 2008, Mastrandrea and Luers 2012). There is a growing body of work on the effects of drought on birds that breed in arid and semiarid habitats. For example, periods of drought or atypically low rainfall have led to lower levels of species richness and abundance in birds (Albright et al. 2010), reduction of birds' reproductive rates (McCreedy et al. 2015), reproductive failure of passerines (Bolger et al. 2005), a decline of over $60 \%$ in density of grassland birds (George et al. 1992), short-term declines in both resident and migratory species (Bock and Bock 1999), and smaller clutch sizes of the California Gnatcatcher (Polioptila californica; Patten and Rotenberry 1999).

Coastal sage scrub is a plant community found in coastal California and northern Baja California, largely below $300 \mathrm{~m}$ in elevation. Its species are adapted to winter rains and a summer dry period, in which many plants are deciduous, growing leaves during the wet winter and losing them during the dry summer. The dominant plant species consist of low-growing shrubs such as Artemisia californica, Salvia mellifera, and Salvia apiana. In this region annual variability in rainfall is high, and fire and drought are recurrent. Shrubs characteristic of coastal sage scrub experience increased mortality following drought (Minnich and Dezzani 1998). Of the numerous species of organisms in coastal sage scrub, approximately 100, both animals and plants, require conservation attention according to California and federal wildlife agencies (Atwood 1993, McCaull 1994). Among birds of coastal southern California, the California Gnatcatcher (designated as threatened by the U.S. Fish and Wildlife Service) and Cactus Wren (Campylorhynchus brunneicapillus), rely on coastal sage scrub for critical breeding habitat (Barr et al. 2015, Rubinoff 
2001). As a result of urban development and fragmentation, this habitat is one of the most endangered in the United States, reduced to less than $10 \%$ of its original range (Taylor 2005), with many species relying on it in decline (Chase et al. 2000, Rubinoff 2001, Barr et al. 2015). The effects of climate change, including longer and more severe droughts, may further threaten southern California's declining, fragmented coastal sage scrub (Griffin and Anchukaitis 2014).

From 2012 to 2016, southern California experienced one of the most severe droughts in recorded history (Griffin and Anchukaitis 2014, Wang et al. 2014, Robeson 2015). The California Department of Water Resources (2015) declared it a "state of emergency" that caused significant economic losses and threatened native wildlife and ecosystems. Protecting and restoring native ecosystems affected by this drought poses a critical conservation challenge. Here, on the basis of long-term data on the bird community in two remnants of coastal sage scrub in Los Angeles County, we consider whether avian species richness changed significantly during drought. We predicted that species richness should decline during drought, especially richness of migratory species that might avoid drought-affected habitat (Albright et al. 2010).

\section{METHODS}

Our study is focused on two patches of coastal sage scrub along the urban-wildland interface of inland Los Angeles County, the Voorhis Ecological Reserve and the Robert J. Bernard Biological Field Station (Figure 1). Precipitation averages $545 \mathrm{~mm}$ per year, although from the year 1900 through 2000 the number of days of rainfall per year decreased (36 to 29), while the average precipitation during each storm increased from 7 to 11 mm (U.S. Geological Survey 2005, Goldstein and Suding 2014). Both the Voorhis Ecological Reserve and the Bernard Field Station have experienced periodic wildfire.

\section{Study Areas}

The Bernard Field Station is located in Claremont on an alluvial outwash from the San Gabriel Mountains (Figure 1; 34.11 ${ }^{\circ} \mathrm{N}, 117.71^{\circ} \mathrm{W}$, elevation $356 \mathrm{~m}$ ). It covers approximately 35 ha and contains an artificial pond and wetland that are managed as a source of permanent water for wildlife. The wetland consists of 0.2 ha of marsh with water up to $2 \mathrm{~m}$ deep and a 0.4 -ha pond up to $6 \mathrm{~m}$ deep. The pond is kept full, even during dry periods. The dominant plant community consists of coastal sage scrub and alluvial fan sage scrub, with some oak woodland and grassland. The reserve also contains small areas of riparian woodland and artificial vernal pools. The portion in coastal sage scrub is mostly undeveloped, with some areas categorized as "recovering" after 5.5 ha burned in an accidental fire in September 2013 (Wallace Meyer pers. comm.).

The 31-ha Voorhis Ecological Reserve is located in the middle of the San Jose Hills in Pomona $\left(34.06^{\circ} \mathrm{N}, 117.83^{\circ} \mathrm{W}\right.$, elevation $300 \mathrm{~m}$ ) approximately $22.5 \mathrm{~km}$ from the Bernard Field Station. It is an important wildlife corridor that connects the San Gabriel and Santa Ana mountains (Figure 1). 


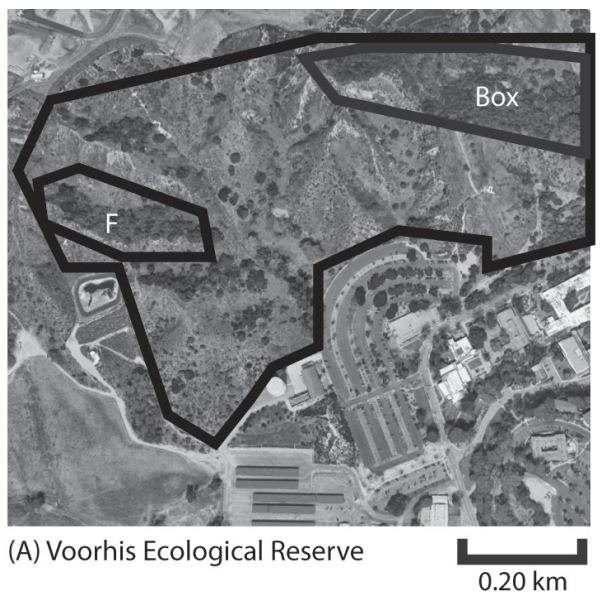

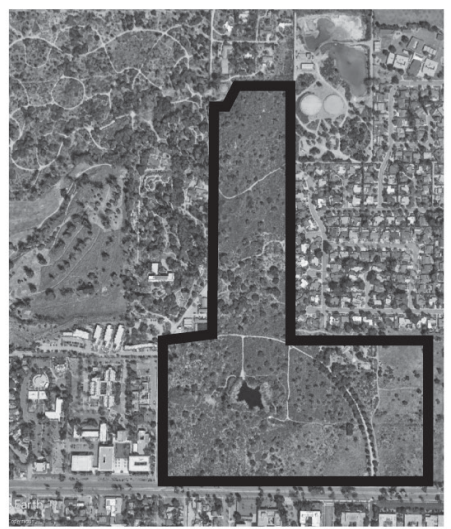

(B) Bernard Field Station

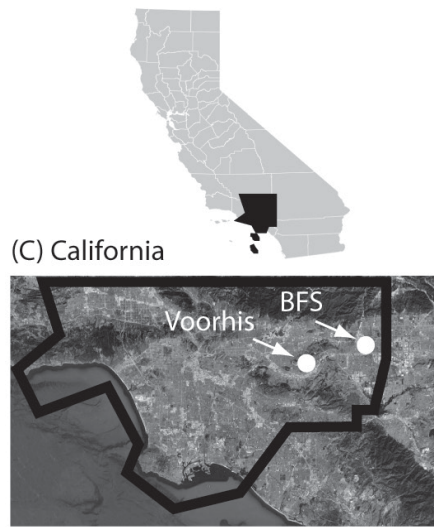

(D) Los Angeles County

$0.30 \mathrm{~km}$

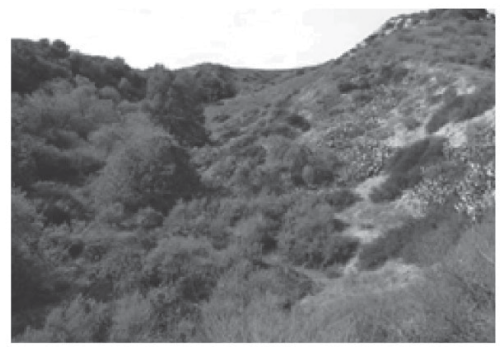

(E) F Canyon

Figure 1. Study areas in Los Angeles County, California (C, D; from Google Earth). (A) Voorhis Ecological Reserve, Pomona (34.059, -117.828, elevation $300 \mathrm{~m}$ ). Polygons indicate Box Canyon and F Canyon survey sites. (B) Bernard Field Station, Claremont $(34.110,-117.710$, elevation $356 \mathrm{~m})$. The permanent lake is shown in the center of the area. (E) View of F Canyon in the Voorhis Ecological Reserve, showing typical vegetation patterns. 
The Voorhis Reserve contains undeveloped coastal sage scrub and has been designated as a "significant ecological area" by Los Angeles County. Like the Bernard Field Station, the Voorhis Reserve is dominated by coastal sage scrub and mixed oak woodland, although the Voorhis Reserve also includes invasive annual plants and some chaparral species. It lacks permanent water but is adjacent to areas with artificial ponds and watered landscaping. The distance to the nearest water source is $0.55 \mathrm{~km}$. The reserve has burned several times, most recently in 1981 (a portion of the reserve) and 1989 (the entire reserve). The 1989 fire was limited to the Voorhis Reserve and small adjacent areas; it did not significantly alter the composition of the reserve's bird community (Moriarty et al. 1985, Moriarty 2013).

\section{Sampling}

The results of monthly bird surveys of the Bernard Field Station by Catherine McFadden from 2000 to 2014 were recorded at www.ebird. org, from which we extracted these data. She completed a four-hour survey over the same route around the perimeter of the 35-ha site on each date (Catherine McFadden and Wallace Meyer pers. comm.). We used only presence/absence data to ensure comparability with results from the Voorhis Ecological Reserve.

From the Voorhis Reserve we used data by Moriarty (2013) based on 265 surveys from January 1983 to December 2003. Each survey covered two canyons (Box Canyon and F Canyon) from a fixed location in each canyon at approximately sunrise for 30 minutes each; which canyon was surveyed first each day alternated. In each canyon the area surveyed was approximately $1.25 \mathrm{ha}, 0.4 \mathrm{~km}$ apart, south-facing, and similar in vegetation structure. According to the protocol of Moriarty (2013), species within the boundaries of the canyon were recorded as present, but birds flying above the canyon to another location were not counted. During a year of exceptional drought, approximately twice per week year round from February 2013 to February 2014, we replicated these surveys from nearly the same positions as Moriarty (2013), for a total of 89 surveys. We followed the same protocol, except in Box Canyon our survey point was about $9 \mathrm{~m}$ above that of Moriarty because recent growth of vegetation impaired visibility from the previous survey point. The large number of these surveys allowed us to examine how drought altered community diversity by month rather than just by season. Both sites were surveyed at the same time, allowing us to compare their species richness.

\section{Classification}

Following Moriarty (2013), we categorized the surveys as summer (April through September) or winter (October through March), defining the seasons by the most significant differences observed in the presence and absence of migratory birds. We classified each species as a year-round resident, transient, or migrant, and separated migrants into two subcategories, summer visitors and winter visitors. Migratory birds were classified as summer visitors if found within or near our field sites during the summer season and classified as winter visitors if normally observed within or near our field sites during the winter season, on the basis of information provided in the Los Angeles 
Breeding Bird Atlas (Allen et al. 1994) and occurrence records at www.eBird. org). We categorized species as breeding at our sites (resident or summer) if recorded adjacent to or within atlas block "SAD" in Allen et al. (1994). Further descriptions within each species account assisted with classification as resident or summer breeding birds. For species that were ambiguous, not listed in the atlas, or for those that breed in habitats beyond our study sites, we first consulted Allen et al. (1994), then eBird for the time of year in which a species was observed most often. This was necessary for several species, including the Pacific-slope Flycatcher (Empidonax difficilis), Townsend's Warbler (Setophaga townsendi), and Phainopepla (Phainopepla nitens).

The distributions of some species changed through the course of our study, as in the cases of the Allen's Hummingbird (Selasphorus sasin), Eurasian Collared-Dove (Streptopelia decaocto), Dark-eyed Junco (Junco hyemalis), and Mountain Chickadee (Poecile gambeli). For these species, which have all recently forged their way into our study areas, we based categorizations on current distributions and patterns, because these species were either never observed in any survey at either site before they colonized the area and became residents (Eurasian Collared-Dove, Allen's Hummingbird), or were observed with the same regularity at our study sites before and after their expansion (Eurasian Collared-Dove and Mountain Chickadee; eBird). We categorized birds as transient if they were observed only intermittently passing through a site during migration. Our use of "migrants" unqualified encompasses summer visitors and winter visitors combined. We included only birds using coastal sage scrub, excluding those found in other habitats at the Bernard Field Station, to ensure that comparisons with the Voorhis Reserve were meaningful.

\section{Drought}

To quantify the effect of drought, we adapted the Palmer Drought Index, based on monthly departures of precipitation and temperature data from long-term averages (Palmer 1965). Values of -4 and below represent "extreme drought," -3 to -3.99 "severe drought," -2 to -2.99 "moderate drought," -1.99 to 2.99 "mid-range," 2-2.99 "moderately moist" conditions, 3-3.99 "very moist," and 3-3.99 "extremely moist." The Palmer Drought Index is a widely used way to analyze fluctuations in drought data over time (Palmer 1965, NOAA 2017). To simplify the index, we reduced the values to categories, then ranked them on a scale from 0 to 6,0 for extremely moist, 1 for very moist, 2 for moderately moist, 3 for mid-range, 4 for moderate drought, 5 for severe drought, and 6 for extreme drought.

\section{Data Analysis}

We used three datasets to examine the effects of drought and season on species richness: (1) long-term data from the Bernard Field Station, 20002014, (2) long-term data from the Voorhis Reserve, 1983-2003, and (3) 2013-2014 data from both sites. For the Voorhis Reserve we pooled results from the two canyons for each date to generate a single list of species. We tested separate models for the following dependent variables: total richness of bird species, richness of resident species, and richness of migratory species in each long-term dataset (1 and 2). At both sites the sampling on which 
the long-term data were based was not consistent from month to month. To standardize the data for variation in sampling effort, we calculated the mean species richness over all sampling dates for each month, then used the value for each month as one data point.

We used general linear mixed models to test the effects of the factors drought level (adapted Palmer Index), season, and year on species richness from the two long-term datasets. We included year as a random variable; all other factors were fixed. We also included the interaction term season $x$ drought level because drought might have a greater effect on the community in winter or summer. We tested the models with the data from the two sites separately and log-transformed data when needed to improve the normality and homogeneity of variance. We analyzed all models in $\mathrm{R}$ version 3.4.3 (R Core Team 2015).

\section{RESULTS}

The numbers of species of year-round residents, winter migrants, summer migrants, transients, and all categories pooled were higher at the Bernard Field Station than at the Voorhis Reserve (Figure 2). The 93 species observed from 2013 to 2014 at the Bernard Field Station comprised 48 resident species, 29 migrants (16 winter visitors, 13 summer visitors), and 16 transient species. The 66 species observed over the same interval at the Voorhis Reserve encompassed 41 residents, 20 migrants (8 winter visitors and 12 summer visitors), and 5 transient species. Ninety-nine species were observed over 265 surveys from 1983 to 2003 in the Voorhis Reserve (55 residents, 12 winter visitors, 11 summer visitors, and 21 transients); 122 species were observed over 160 surveys from 2000 to 2014 at the Bernard Field Station (62 residents, 16 winter visitors, 14 summer visitors, and 30 transients). Several species, both residents and migrants, at both the Bernard Field Station and the Voorhis Reserve, were observed during surveys before the drought but not after the drought (Table 1).

\section{Species Richness}

At the Voorhis Reserve, total species richness varied by season, being higher in summer than in winter (Table 2; significant effect of season). There were no other significant factors affecting richness of total species at either study site, although the effect of drought level on richness of resident species was significant at both sites (Table 2). The number of resident species was higher during wet conditions (drought indices 0-2) and lower during drought conditions (drought indices 4-6), with the trend more pronounced at the Voorhis Reserve than at the Bernard Field Station (Figure 3a, d). The number of migrant species recorded at the Voorhis Reserve did not differ significantly by drought level, whereas at the Bernard Field Station it was higher during drought (index classes 5 and 6) than during wetter conditions (Table 2, Figure 3e). The number of migrant species was higher in summer than in winter at both sites (Table 2, significant effect of season, Figure 3b, e). Overall, richness of resident species was higher during wetter periods than during drought at both study sites, while that of migrant species at the Voorhis Reserve in drought and nondrought periods did not differ. 

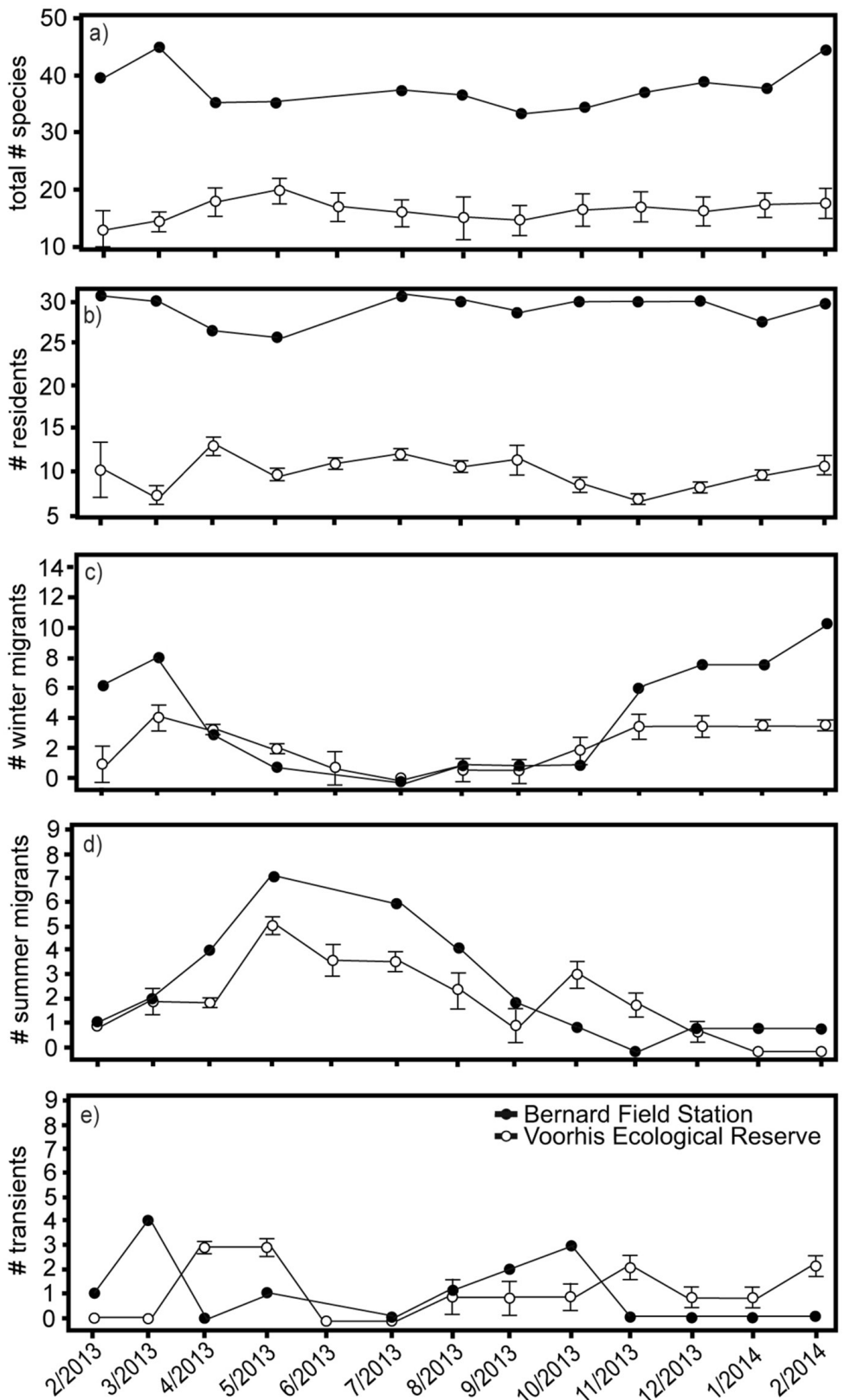

Figure 2. Number of bird species recorded at the Voorhis Ecological Reserve and Bernard Field Station by month from February 2013 to February 2014: (a) total species richness; (b) richness of resident species; (c) species richness of winter visitors; (d) species richness of summer visitors; (e) richness of transient species. Symbols indicate group means; error bars are one standard error. There are no error bars for Bernard Field Station data because each point represents one instance of sampling. 

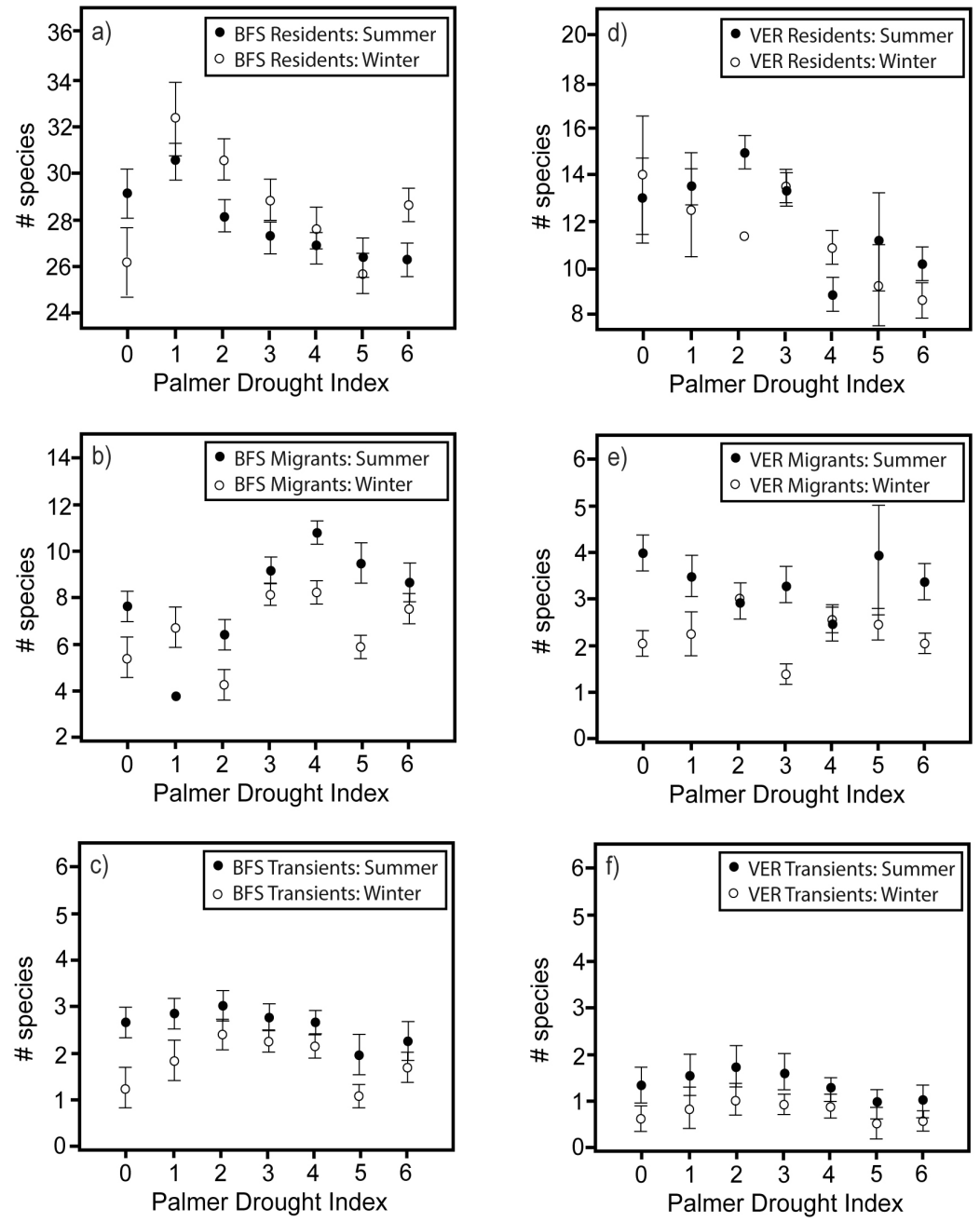

Figure 3. Bird species richness by drought index and season (a) for resident species from 2000 to 2014 at the Bernard Field Station (BFS), (b) for all migrant species from 2000 to 2014 in the Bernard Field Station, (c) for transient species from 1983 to 2003 at the Bernard Field Station, (d) for resident species from 1983 to 2003 at the Voorhis Ecological Reserve (VER), (e) for all migrant species from 1983 to 2003 at the Voorhis Ecological Reserve, and (f) for all transient species from 1983 to 2003 at the Voorhis Ecological Reserve. Palmer drought index scores of 4-6 indicate drought conditions. Symbols indicate group means; error bars are one standard error. 
Table 1 Categorization of Status and Occurrence of Birds before or during Drought at the Bernard Field Station and Voorhis Ecological Reserve, Southern California, 1981-2014

\begin{tabular}{|c|c|c|c|c|c|}
\hline \multirow[b]{2}{*}{ Species } & \multirow[b]{2}{*}{ Status } & \multicolumn{2}{|c|}{ Bernard } & \multicolumn{2}{|c|}{ Voorhis } \\
\hline & & Pre-drought & Drought & Pre-drought & Drought \\
\hline $\begin{array}{l}\text { California Quail (Callipepla } \\
\text { californica) }\end{array}$ & Resident & $\mathrm{X}$ & $\mathrm{X}$ & $\mathrm{X}$ & $\mathrm{X}$ \\
\hline Rock Pigeon (Columba livia) & Resident & $\mathrm{X}$ & $\mathrm{X}$ & - & - \\
\hline $\begin{array}{l}\text { Band-tailed Pigeon (Patagioenas } \\
\text { fasciata) }\end{array}$ & Resident & $\mathrm{X}$ & $\mathrm{X}$ & $\mathrm{X}$ & - \\
\hline $\begin{array}{l}\text { Eurasian Collared-Dove } \\
\quad \text { (Streptopelia decaocto) }\end{array}$ & Resident & $\mathrm{X}$ & - & - & - \\
\hline $\begin{array}{l}\text { Spotted Dove (Streptopelia } \\
\text { chinensis) }\end{array}$ & Resident & - & - & $\mathrm{X}$ & - \\
\hline $\begin{array}{l}\text { Mourning Dove (Zenaida } \\
\text { macroura) }\end{array}$ & Resident & $\mathrm{X}$ & $\mathrm{X}$ & $\mathrm{X}$ & $\mathrm{X}$ \\
\hline $\begin{array}{l}\text { Greater Roadrunner (Geococcyx } \\
\text { californianus) }\end{array}$ & Resident & $\mathrm{X}$ & - & $\mathrm{X}$ & - \\
\hline $\begin{array}{l}\text { Common Poorwill } \\
\text { (Phalaenoptilus nuttallii) }\end{array}$ & Transient & - & - & $\mathrm{X}$ & - \\
\hline Vaux's Swift (Chaetura vauxi) & Transient & $X$ & $\mathrm{X}$ & $X$ & - \\
\hline $\begin{array}{l}\text { White-throated Swift } \\
\text { (Aeronautes saxatalis) }\end{array}$ & Resident & $\mathrm{X}$ & $X$ & $X$ & - \\
\hline $\begin{array}{l}\text { Black-chinned Hummingbird } \\
\text { (Archilochus alexandri) }\end{array}$ & Summer & $\mathrm{X}$ & $\mathrm{X}$ & $\mathrm{X}$ & $\mathrm{X}$ \\
\hline $\begin{array}{l}\text { Anna's Hummingbird (Calypte } \\
\text { anna) }\end{array}$ & Resident & $\mathrm{X}$ & $\mathrm{X}$ & $\mathrm{X}$ & $\mathrm{X}$ \\
\hline $\begin{array}{l}\text { Costa's Hummingbird (Calypte } \\
\text { costae) }\end{array}$ & Summer & $\mathrm{X}$ & $\mathrm{X}$ & $\mathrm{X}$ & $\mathrm{X}$ \\
\hline $\begin{array}{l}\text { Rufous Hummingbird } \\
\text { (Selasphorus rufus) }\end{array}$ & Transient & $\mathrm{X}$ & $\mathrm{X}$ & $\mathrm{X}$ & - \\
\hline $\begin{array}{l}\text { Allen's Hummingbird } \\
\text { (Selasphorus sasin) }\end{array}$ & Resident & $\mathrm{X}$ & $\mathrm{X}$ & $\mathrm{X}$ & $\mathrm{X}$ \\
\hline $\begin{array}{l}\text { Calliope Hummingbird } \\
\text { (Selasphorus calliope) }\end{array}$ & Transient & $\mathrm{X}$ & - & - & - \\
\hline $\begin{array}{l}\text { Great Blue Heron (Ardea } \\
\text { herodias) }\end{array}$ & Resident & $\mathrm{X}$ & - & - & $\mathrm{X}$ \\
\hline Turkey Vulture (Cathartes aura) & Resident & $\mathrm{X}$ & $\mathrm{X}$ & $\mathrm{X}$ & $\mathrm{X}$ \\
\hline $\begin{array}{l}\text { White-tailed Kite (Elanus } \\
\text { leucurus) }\end{array}$ & Resident & $\mathrm{X}$ & - & $\mathrm{X}$ & - \\
\hline $\begin{array}{l}\text { Northern Harrier (Circus } \\
\text { hudsonius) }\end{array}$ & Resident & $\mathrm{X}$ & - & $\mathrm{X}$ & $\mathrm{X}$ \\
\hline $\begin{array}{l}\text { Sharp-shinned Hawk (Accipiter } \\
\text { striatus) }\end{array}$ & Winter & $\mathrm{X}$ & $\mathrm{X}$ & $\mathrm{X}$ & $\mathrm{X}$ \\
\hline $\begin{array}{l}\text { Cooper's Hawk (Accipiter } \\
\text { cooperii) }\end{array}$ & Resident & $\mathrm{X}$ & $\mathrm{X}$ & $\mathrm{X}$ & $\mathrm{X}$ \\
\hline $\begin{array}{l}\text { Red-shouldered Hawk (Buteo } \\
\text { lineatus) }\end{array}$ & Resident & $\mathrm{X}$ & $\mathrm{X}$ & - & $\mathrm{X}$ \\
\hline $\begin{array}{l}\text { Swainson's Hawk (Buteo } \\
\text { swainsoni) }\end{array}$ & Transient & $\mathrm{X}$ & - & - & - \\
\hline $\begin{array}{l}\text { Red-tailed Hawk (Buteo } \\
\text { jamaicensis) }\end{array}$ & Resident & $\mathrm{X}$ & $\mathrm{X}$ & $\mathrm{X}$ & $\mathrm{X}$ \\
\hline
\end{tabular}




\begin{tabular}{|c|c|c|c|c|c|}
\hline \multirow[b]{2}{*}{ Species } & \multirow[b]{2}{*}{ Status } & \multicolumn{2}{|c|}{ Bernard } & \multicolumn{2}{|c|}{ Voorhis } \\
\hline & & Pre-drought & Drought & Pre-drought & Drought \\
\hline $\begin{array}{l}\text { Golden Eagle (Aquila } \\
\text { chrysaetos) }\end{array}$ & Transient & - & - & $\mathrm{X}$ & - \\
\hline Barn Owl (Tyto alba) & Resident & $\mathrm{X}$ & - & - & - \\
\hline $\begin{array}{l}\text { Great Horned Owl } \\
\text { (Bubo virginianus) }\end{array}$ & Resident & $\mathrm{X}$ & $\mathrm{X}$ & $\mathrm{X}$ & $\mathrm{X}$ \\
\hline $\begin{array}{l}\text { Acorn Woodpecker } \\
\text { (Melanerpes formicivorus) }\end{array}$ & Resident & $\mathrm{X}$ & $\mathrm{X}$ & $\mathrm{X}$ & - \\
\hline $\begin{array}{l}\text { Red-breasted Sapsucker } \\
\text { (Sphyrapicus ruber) }\end{array}$ & Winter & $\mathrm{X}$ & $\mathrm{X}$ & - & - \\
\hline $\begin{array}{l}\text { Nuttall's Woodpecker } \\
\text { (Dryobates nuttallii) }\end{array}$ & Resident & $\mathrm{X}$ & $\mathrm{X}$ & $\mathrm{X}$ & $\mathrm{X}$ \\
\hline $\begin{array}{l}\text { Downy Woodpecker } \\
\text { (Dryobates pubescens) }\end{array}$ & Resident & $\mathrm{X}$ & $\mathrm{X}$ & - & - \\
\hline $\begin{array}{l}\text { Northern Flicker (Colaptes } \\
\text { auratus) }\end{array}$ & Resident & $\mathrm{X}$ & $\mathrm{X}$ & $\mathrm{X}$ & $\mathrm{X}$ \\
\hline $\begin{array}{l}\text { American Kestrel } \\
\text { (Falco sparverius) }\end{array}$ & Resident & $\mathrm{X}$ & $\mathrm{X}$ & $\mathrm{X}$ & $\mathrm{X}$ \\
\hline Merlin (Falco columbarius) & Winter & $\mathrm{X}$ & $\mathrm{X}$ & - & - \\
\hline $\begin{array}{l}\text { Prairie Falcon (Falco } \\
\text { mexicanus) }\end{array}$ & Transient & - & - & $\mathrm{X}$ & - \\
\hline $\begin{array}{l}\text { Red-crowned Parrot } \\
\text { (Amazona viridigenalis) }\end{array}$ & Resident & $\mathrm{X}$ & - & - & - \\
\hline $\begin{array}{l}\text { Olive-sided Flycatcher } \\
\text { (Contopus cooperi) }\end{array}$ & Transient & $\mathrm{X}$ & $\mathrm{X}$ & - & - \\
\hline $\begin{array}{l}\text { Western Wood-Pewee } \\
\text { (Contopus sordidulus) }\end{array}$ & Transient & $\mathrm{X}$ & - & $\mathrm{X}$ & - \\
\hline $\begin{array}{l}\text { Willow Flycatcher (Empidonax } \\
\text { traillii) }\end{array}$ & Transient & $\mathrm{X}$ & $\mathrm{X}$ & - & - \\
\hline $\begin{array}{l}\text { Hammond's Flycatcher } \\
\text { (Empidonax hammondii) }\end{array}$ & Transient & $\mathrm{X}$ & $\mathrm{X}$ & - & - \\
\hline $\begin{array}{l}\text { Gray Flycatcher (Empidonax } \\
\text { wrightii) }\end{array}$ & Transient & $\mathrm{X}$ & - & $\mathrm{X}$ & - \\
\hline $\begin{array}{l}\text { Pacific-slope Flycatcher } \\
\text { (Empidonax difficilis) }\end{array}$ & Summer & $\mathrm{X}$ & - & $\mathrm{X}$ & $\mathrm{X}$ \\
\hline $\begin{array}{l}\text { Black Phoebe (Sayornis } \\
\text { nigricans) }\end{array}$ & Resident & $\mathrm{X}$ & $\mathrm{X}$ & $\mathrm{X}$ & $\mathrm{X}$ \\
\hline Say's Phoebe (Sayornis saya) & Resident & $\mathrm{X}$ & $\mathrm{X}$ & $\mathrm{X}$ & $\mathrm{X}$ \\
\hline $\begin{array}{l}\text { Ash-throated Flycatcher } \\
\text { (Myiarchus cinerascens) }\end{array}$ & Summer & $\mathrm{X}$ & $\mathrm{X}$ & $\mathrm{X}$ & $\mathrm{X}$ \\
\hline $\begin{array}{l}\text { Cassin's Kingbird (Tyrannus } \\
\text { vociferans) }\end{array}$ & Resident & $\mathrm{X}$ & $\mathrm{X}$ & $\mathrm{X}$ & $\mathrm{X}$ \\
\hline $\begin{array}{l}\text { Western Kingbird (Tyrannus } \\
\text { verticalis) }\end{array}$ & Summer & $\mathrm{X}$ & $\mathrm{X}$ & $\mathrm{X}$ & $\mathrm{X}$ \\
\hline $\begin{array}{l}\text { Loggerhead Shrike (Lanius } \\
\text { ludovicianus) }\end{array}$ & Resident & $\mathrm{X}$ & - & $\mathrm{X}$ & $\mathrm{X}$ \\
\hline Hutton's Vireo (Vireo huttoni) & Resident & $\mathrm{X}$ & $\mathrm{X}$ & - & - \\
\hline Cassin's Vireo (Vireo cassinii) & Transient & $\mathrm{X}$ & $\mathrm{X}$ & - & $\mathrm{X}$ \\
\hline $\begin{array}{l}\text { Plumbeous Vireo (Vireo } \\
\text { plumbeus) }\end{array}$ & Transient & - & - & $X$ & - \\
\hline Warbling Vireo (Vireo gilvus) & Transient & $\mathrm{X}$ & - & $X$ & - \\
\hline
\end{tabular}


Table 1 (continued).

\begin{tabular}{|c|c|c|c|c|c|}
\hline \multirow[b]{2}{*}{ Species } & \multirow[b]{2}{*}{ Status } & \multicolumn{2}{|c|}{ Bernard } & \multicolumn{2}{|c|}{ Voorhis } \\
\hline & & Pre-drought & Drought & Pre-drought & Drought \\
\hline Bell's Vireo (Vireo bellii) & Summer & $\mathrm{X}$ & - & - & - \\
\hline $\begin{array}{l}\text { California Scrub-Jay } \\
\text { (Aphelocoma californica) }\end{array}$ & Resident & $\mathrm{x}$ & $\mathrm{x}$ & $\mathrm{X}$ & $\mathrm{x}$ \\
\hline $\begin{array}{l}\text { American Crow (Corvus } \\
\text { brachyrhynchos) }\end{array}$ & Resident & $\mathrm{X}$ & $\mathrm{x}$ & $\mathrm{x}$ & $\mathrm{x}$ \\
\hline Common Raven (Corvus corax) & Resident & $\mathrm{X}$ & $\mathrm{X}$ & $\mathrm{X}$ & $\mathrm{X}$ \\
\hline $\begin{array}{l}\text { Tree Swallow (Tachycineta } \\
\text { bicolor) }\end{array}$ & Transient & $\mathrm{X}$ & - & - & - \\
\hline $\begin{array}{l}\text { Violet-green Swallow } \\
\text { (Tachycineta thalassina) }\end{array}$ & Transient & $\mathrm{X}$ & $\mathrm{x}$ & $\mathrm{X}$ & - \\
\hline $\begin{array}{l}\text { Northern Rough-winged } \\
\text { Swallow (Stelgidopteryx } \\
\text { serripennis) }\end{array}$ & Summer & $\mathrm{X}$ & $\mathrm{X}$ & $\mathrm{x}$ & $\mathrm{X}$ \\
\hline $\begin{array}{l}\text { Cliff Swallow (Petrochelidon } \\
\text { pyrrhonota) }\end{array}$ & Summer & $\mathrm{X}$ & $\mathrm{x}$ & $\mathrm{x}$ & $\mathrm{x}$ \\
\hline Barn Swallow (Hirundo rustica) & Summer & $\mathrm{X}$ & $\mathrm{X}$ & - & - \\
\hline $\begin{array}{l}\text { Mountain Chickadee (Poecile } \\
\text { gambeli) }\end{array}$ & Transient & $\mathrm{x}$ & $\mathrm{x}$ & $\mathrm{x}$ & $\mathrm{x}$ \\
\hline $\begin{array}{l}\text { Oak Titmouse (Baeolophus } \\
\text { inornatus) }\end{array}$ & Resident & $\mathrm{X}$ & $\mathrm{x}$ & $\mathrm{X}$ & - \\
\hline Bushtit (Psaltriparus minimus) & Resident & $\mathrm{X}$ & $\mathrm{X}$ & $\mathrm{X}$ & $\mathrm{X}$ \\
\hline $\begin{array}{l}\text { White-breasted Nuthatch (Sitta } \\
\text { carolinensis) }\end{array}$ & Transient & $\mathrm{X}$ & $\mathrm{x}$ & - & - \\
\hline $\begin{array}{l}\text { Rock Wren (Salpinctes } \\
\text { obsoletus) }\end{array}$ & Resident & $\mathrm{x}$ & - & $\mathrm{x}$ & - \\
\hline $\begin{array}{l}\text { House Wren (Troglodytes } \\
\text { aedon) }\end{array}$ & Resident & $\mathrm{x}$ & $\mathrm{x}$ & $\mathrm{x}$ & $\mathrm{x}$ \\
\hline $\begin{array}{l}\text { Bewick's Wren (Thryomanes } \\
\text { bewickii) }\end{array}$ & Resident & $\mathrm{X}$ & $\mathrm{x}$ & $\mathrm{X}$ & $\mathrm{x}$ \\
\hline $\begin{array}{l}\text { Cactus Wren (Campylorhynchus } \\
\text { brunneicapillus) }\end{array}$ & Resident & $\mathrm{x}$ & - & $\mathrm{x}$ & $\mathrm{x}$ \\
\hline $\begin{array}{l}\text { Blue-gray Gnatcatcher } \\
\quad \text { (Polioptila caerulea) }\end{array}$ & Resident & $\mathrm{x}$ & $\mathrm{x}$ & $\mathrm{x}$ & $\mathrm{x}$ \\
\hline $\begin{array}{l}\text { California Gnatcatcher } \\
\text { (Polioptila californica) }\end{array}$ & Resident & - & - & $\mathrm{x}$ & $\mathrm{x}$ \\
\hline $\begin{array}{l}\text { Ruby-crowned Kinglet (Regulus } \\
\text { calendula) }\end{array}$ & Winter & $\mathrm{X}$ & $\mathrm{x}$ & $\mathrm{x}$ & $\mathrm{x}$ \\
\hline Wrentit (Chamaea fasciata) & Resident & $\mathrm{X}$ & $\mathrm{X}$ & $\mathrm{x}$ & $\mathrm{x}$ \\
\hline $\begin{array}{l}\text { Western Bluebird (Sialia } \\
\text { mexicana) }\end{array}$ & Resident & $\mathrm{x}$ & $\mathrm{x}$ & $\mathrm{X}$ & X \\
\hline $\begin{array}{l}\text { Swainson's Thrush (Catharus } \\
\text { ustulatus) }\end{array}$ & Transient & $\mathrm{X}$ & - & $\mathrm{X}$ & $\mathrm{X}$ \\
\hline $\begin{array}{l}\text { Hermit Thrush (Catharus } \\
\text { guttatus) }\end{array}$ & Winter & $\mathrm{X}$ & $\mathrm{X}$ & $\mathrm{X}$ & $\mathrm{X}$ \\
\hline $\begin{array}{l}\text { American Robin (Turdus } \\
\text { migratorius) }\end{array}$ & Resident & $\mathrm{X}$ & $\mathrm{X}$ & $\mathrm{X}$ & $\mathrm{X}$ \\
\hline $\begin{array}{l}\text { California Thrasher (Toxostoma } \\
\text { redivivum) }\end{array}$ & Resident & $\mathrm{X}$ & $\mathrm{X}$ & $\mathrm{X}$ & $\mathrm{x}$ \\
\hline $\begin{array}{l}\text { Northern Mockingbird (Mimus } \\
\text { polyglottos) }\end{array}$ & Resident & $\mathrm{X}$ & $\mathrm{x}$ & $\mathrm{X}$ & $\mathrm{X}$ \\
\hline
\end{tabular}




\begin{tabular}{|c|c|c|c|c|c|}
\hline \multirow[b]{2}{*}{ Species } & \multirow[b]{2}{*}{ Status } & \multicolumn{2}{|c|}{ Bernard } & \multicolumn{2}{|c|}{ Voorhis } \\
\hline & & Pre-drought & Drought & Pre-drought & Drought \\
\hline $\begin{array}{l}\text { European Starling (Sturnus } \\
\text { vulgaris) }\end{array}$ & Resident & $\mathrm{X}$ & $\mathrm{X}$ & $\mathrm{X}$ & - \\
\hline $\begin{array}{l}\text { Cedar Waxwing (Bombycilla } \\
\text { cedrorum) }\end{array}$ & Winter & $\mathrm{X}$ & $\mathrm{X}$ & $\mathrm{X}$ & - \\
\hline $\begin{array}{l}\text { Phainopepla (Phainopepla } \\
\text { nitens) }\end{array}$ & Summer & $\mathrm{X}$ & $\mathrm{X}$ & $\mathrm{X}$ & $\mathrm{X}$ \\
\hline $\begin{array}{l}\text { Northern Red Bishop } \\
\quad \text { (Euplectes franciscanus) }\end{array}$ & Resident & $\mathrm{X}$ & - & - & - \\
\hline $\begin{array}{l}\text { Scaly-breasted Munia } \\
\quad \text { (Lonchura punctulata) }\end{array}$ & Resident & $\mathrm{X}$ & - & - & - \\
\hline $\begin{array}{l}\text { House Sparrow (Passer } \\
\text { domesticus) }\end{array}$ & Resident & $\mathrm{X}$ & $\mathrm{X}$ & $\mathrm{X}$ & - \\
\hline $\begin{array}{l}\text { House Finch (Haemorhous } \\
\text { mexicanus) }\end{array}$ & Resident & $\mathrm{X}$ & $\mathrm{X}$ & $\mathrm{X}$ & $\mathrm{X}$ \\
\hline $\begin{array}{l}\text { Purple Finch (Haemorhous } \\
\text { purpureus) }\end{array}$ & Winter & $\mathrm{X}$ & $\mathrm{X}$ & $\mathrm{X}$ & - \\
\hline Pine Siskin (Spinus pinus) & Winter & $\mathrm{X}$ & $\mathrm{X}$ & - & - \\
\hline $\begin{array}{l}\text { Lesser Goldfinch (Spinus } \\
\text { psaltria) }\end{array}$ & Resident & $\mathrm{X}$ & $\mathrm{X}$ & $\mathrm{X}$ & $X$ \\
\hline $\begin{array}{l}\text { Lawrence's Goldfinch (Spinus } \\
\text { lawrencei) }\end{array}$ & Transient & $\mathrm{X}$ & $\mathrm{X}$ & $\mathrm{X}$ & - \\
\hline $\begin{array}{l}\text { American Goldfinch (Spinus } \\
\text { tristis) }\end{array}$ & Resident & $\mathrm{X}$ & $\mathrm{X}$ & $\mathrm{X}$ & $\mathrm{X}$ \\
\hline $\begin{array}{l}\text { Spotted Towhee (Pipilo } \\
\text { maculatus) }\end{array}$ & Resident & $\mathrm{X}$ & $\mathrm{X}$ & $\mathrm{X}$ & $\mathrm{X}$ \\
\hline $\begin{array}{l}\text { Rufous-crowned Sparrow } \\
\text { (Aimophila ruficeps) }\end{array}$ & Resident & - & - & $\mathrm{X}$ & $\mathrm{X}$ \\
\hline $\begin{array}{l}\text { California Towhee (Melozone } \\
\text { crissalis) }\end{array}$ & Resident & $\mathrm{X}$ & $\mathrm{X}$ & $\mathrm{X}$ & $\mathrm{X}$ \\
\hline $\begin{array}{l}\text { Chipping Sparrow (Spizella } \\
\text { passerina) }\end{array}$ & Winter & $\mathrm{X}$ & $\mathrm{X}$ & $\mathrm{X}$ & $\mathrm{X}$ \\
\hline $\begin{array}{l}\text { Brewer's Sparrow (Spizella } \\
\text { breweri) }\end{array}$ & Transient & $\mathrm{X}$ & $\mathrm{X}$ & - & - \\
\hline $\begin{array}{l}\text { Lark Sparrow (Chondestes } \\
\text { grammacus) }\end{array}$ & Resident & $X$ & $X$ & $X$ & - \\
\hline $\begin{array}{l}\text { Bell's Sparrow (Artemisiospiza } \\
\text { belli) }\end{array}$ & Resident & - & - & $\mathrm{X}$ & - \\
\hline $\begin{array}{l}\text { Savannah Sparrow (Passerculus } \\
\text { sandwichensis) }\end{array}$ & Transient & $\mathrm{X}$ & $\mathrm{X}$ & $\mathrm{X}$ & - \\
\hline Fox Sparrow (Passerella iliaca) & Winter & $\mathrm{X}$ & $\mathrm{X}$ & $\mathrm{X}$ & - \\
\hline $\begin{array}{l}\text { Song Sparrow (Melospiza } \\
\text { melodia) }\end{array}$ & Resident & $\mathrm{X}$ & $\mathrm{X}$ & $\mathrm{X}$ & $\mathrm{X}$ \\
\hline $\begin{array}{l}\text { Lincoln's Sparrow (Melospiza } \\
\text { lincolnii) }\end{array}$ & Winter & $\mathrm{X}$ & $\mathrm{X}$ & $\mathrm{X}$ & - \\
\hline $\begin{array}{l}\text { White-throated Sparrow } \\
\text { (Zonotrichia albicollis) }\end{array}$ & Winter & $X$ & $\mathrm{X}$ & - & - \\
\hline $\begin{array}{l}\text { White-crowned Sparrow } \\
\text { (Zonotrichia leucophrys) }\end{array}$ & Winter & $\mathrm{X}$ & $\mathrm{X}$ & $\mathrm{X}$ & $\mathrm{X}$ \\
\hline $\begin{array}{l}\text { Golden-crowned Sparrow } \\
\text { (Zonotrichia atricapilla) }\end{array}$ & Winter & $\mathrm{X}$ & $\mathrm{X}$ & $\mathrm{X}$ & $X$ \\
\hline
\end{tabular}


Table 1 (continued).

\begin{tabular}{|c|c|c|c|c|c|}
\hline \multirow[b]{2}{*}{ Species } & \multirow[b]{2}{*}{ Status } & \multicolumn{2}{|c|}{ Bernard } & \multicolumn{2}{|c|}{ Voorhis } \\
\hline & & Pre-drought & Drought & Pre-drought & Drought \\
\hline $\begin{array}{l}\text { Dark-eyed Junco (Junco } \\
\text { hyemalis) }\end{array}$ & Winter & $\mathrm{X}$ & $\mathrm{X}$ & $\mathrm{X}$ & $\mathrm{X}$ \\
\hline $\begin{array}{l}\text { Yellow-breasted Chat (Icteria } \\
\text { virens) }\end{array}$ & Summer & $\mathrm{X}$ & $\mathrm{X}$ & - & - \\
\hline $\begin{array}{l}\text { Western Meadowlark (Sturnella } \\
\text { neglecta) }\end{array}$ & Resident & $\mathrm{X}$ & $\mathrm{X}$ & $\mathrm{X}$ & $\mathrm{X}$ \\
\hline $\begin{array}{l}\text { Hooded Oriole (Icterus } \\
\text { cucullatus) }\end{array}$ & Summer & $\mathrm{X}$ & $\mathrm{X}$ & $\mathrm{X}$ & $\mathrm{X}$ \\
\hline $\begin{array}{l}\text { Bullock's Oriole (Icterus } \\
\text { bullockii) }\end{array}$ & Summer & $\mathrm{X}$ & $\mathrm{X}$ & $\mathrm{X}$ & $\mathrm{X}$ \\
\hline $\begin{array}{l}\text { Red-winged Blackbird (Agelaius } \\
\text { phoeniceus) }\end{array}$ & Resident & $\mathrm{X}$ & $\mathrm{X}$ & $\mathrm{X}$ & - \\
\hline $\begin{array}{l}\text { Brown-headed Cowbird } \\
\text { (Molothrus ater) }\end{array}$ & Resident & $\mathrm{X}$ & $\mathrm{X}$ & X & $\mathrm{X}$ \\
\hline $\begin{array}{l}\text { Brewer's Blackbird (Euphagus } \\
\text { cyanocephalus) }\end{array}$ & Resident & $\mathrm{X}$ & - & - & - \\
\hline $\begin{array}{l}\text { Great-tailed Grackle (Quiscalus } \\
\text { mexicanus) }\end{array}$ & Resident & $\mathrm{X}$ & - & - & - \\
\hline $\begin{array}{l}\text { Orange-crowned Warbler } \\
\text { (Oreothlypis celata) }\end{array}$ & Resident & $\mathrm{X}$ & $\mathrm{X}$ & $\mathrm{X}$ & - \\
\hline $\begin{array}{l}\text { Nashville Warbler (Oreothlypis } \\
\text { ruficapilla) }\end{array}$ & Transient & $\mathrm{X}$ & $\mathrm{X}$ & - & - \\
\hline $\begin{array}{l}\text { MacGillivray's Warbler } \\
\text { (Geothlypis tolmiei) }\end{array}$ & Transient & $\mathrm{X}$ & - & $\mathrm{X}$ & - \\
\hline $\begin{array}{l}\text { Common Yellowthroat } \\
\text { (Geothlypis trichas) }\end{array}$ & Resident & $\mathrm{X}$ & $\mathrm{X}$ & - & - \\
\hline $\begin{array}{l}\text { Yellow Warbler (Setophaga } \\
\text { petechia) }\end{array}$ & Summer & $\mathrm{X}$ & - & $\mathrm{X}$ & $\mathrm{X}$ \\
\hline $\begin{array}{l}\text { Yellow-rumped Warbler } \\
\text { (Setophaga coronata) }\end{array}$ & Winter & $\mathrm{X}$ & $\mathrm{X}$ & $\mathrm{X}$ & $\mathrm{X}$ \\
\hline $\begin{array}{l}\text { Black-throated Gray Warbler } \\
\text { (Setophaga nigrescens) }\end{array}$ & Transient & $\mathrm{X}$ & $\mathrm{X}$ & - & - \\
\hline $\begin{array}{l}\text { Townsend's Warbler (Setophaga } \\
\text { townsendi) }\end{array}$ & Transient & $\mathrm{X}$ & - & $\mathrm{X}$ & - \\
\hline $\begin{array}{l}\text { Hermit Warbler (Setophaga } \\
\text { occidentalis) }\end{array}$ & Transient & $\mathrm{X}$ & - & - & - \\
\hline $\begin{array}{l}\text { Wilson's Warbler (Cardellina } \\
\text { pusilla) }\end{array}$ & Transient & $\mathrm{X}$ & X & X & X \\
\hline $\begin{array}{l}\text { Summer Tanager (Piranga } \\
\text { rubra) }\end{array}$ & Transient & $\mathrm{X}$ & - & - & - \\
\hline $\begin{array}{l}\text { Western Tanager (Piranga } \\
\text { ludoviciana) }\end{array}$ & Transient & $\mathrm{X}$ & $\mathrm{X}$ & $\mathrm{X}$ & $\mathrm{X}$ \\
\hline $\begin{array}{l}\text { Black-headed Grosbeak } \\
\text { (Pheucticus melanocephalus) }\end{array}$ & Summer & $\mathrm{X}$ & $\mathrm{X}$ & $\mathrm{X}$ & $\mathrm{X}$ \\
\hline $\begin{array}{l}\text { Blue Grosbeak (Passerina } \\
\text { caerulea) }\end{array}$ & Summer & - & $\mathrm{X}$ & - & - \\
\hline $\begin{array}{l}\text { Lazuli Bunting (Passerina } \\
\text { amoena) }\end{array}$ & Summer & $\mathrm{X}$ & - & $\mathrm{X}$ & - \\
\hline
\end{tabular}


Table 2 F Statistics from Generalized Linear Mixed Models of Bird Species Richness from 1983 to 2003 at the Voorhis Ecological Reserve and from 2000 to 2014 at the Bernard Field Station, Los Angeles County, California $^{a}$

\begin{tabular}{|c|c|c|c|}
\hline Response variable and site & Drought level & Season & Drought level $\times$ season \\
\hline Degrees of freedom & 6 & 1 & 6 \\
\hline \multicolumn{4}{|l|}{ Total richness } \\
\hline Voorhis & 1.346 & $5.322^{*}$ & 0.847 \\
\hline Bernard $^{b}$ & 2.004 & 0.387 & 1.742 \\
\hline \multicolumn{4}{|l|}{ Richness of residents } \\
\hline Voorhis & $2.418^{*}$ & 0.124 & 1.468 \\
\hline Bernard & $2.455^{*}$ & $9.125^{* *}$ & 1.869 \\
\hline \multicolumn{4}{|l|}{ Richness of migrants } \\
\hline Voorhis $^{b}$ & 1.707 & $18.231^{* * * *}$ & 0.477 \\
\hline Bernard $^{b}$ & 2.055 & $10.865^{\text {*** }}$ & 1.466 \\
\hline \multicolumn{4}{|l|}{ Richness of transients } \\
\hline Voorhis & 2.111 & 0.542 & 1.009 \\
\hline Bernard & 1.057 & 0.238 & 0.955 \\
\hline
\end{tabular}

${ }^{a}$ Levels of significance: ${ }^{*} P<0.05$, ${ }^{* *} P<0.01$; ${ }^{* * *} P<0.001$; error df were 98 for the Voorhis Ecological Reserve and 121 for the Bernard Field Station.

${ }^{b}$ Data were log-transformed to improve normality.

\section{DISCUSSION}

At the Bernard Field Station, where during the drought the total number of species and the numbers of migrant species and of transients were greater than at the Voorhis Reserve, birds had access to a reliable water source. Drought may alter bird communities by depressing the availability of resources, and the effect of drought varies in different communities. Various studies have shown the negative effect drought has on bird occurrence (Faaborg 1982, Herremans 2004, Stracey 2010, Bennett et al. 2014). In a study based on Breeding Bird Survey data from the central U.S., Albright et al. (2010) found the most negative effects of drought in more arid regions, with species migrating to the tropics affected the most. They suggested that the decline of migrants during drought was due to migrants having the option to pass over unfavorable sites and select better locations, although this might vary with the availability and distance of such better habitat, the pull of site fidelity, which is often strong in long-distance migrants (Berthold 2001), and other factors. Both resident and migrant birds vary in how they react to changes in the environment. Some species are readily able to occupy new habitat under adverse conditions, while others are more heavily driven by site fidelity and return to the same locations year to year (Sedgwick 2004, Winter and Hargrove 2004, Barr et al. 2015). Albright et al. (2010) claimed that resident species in semiarid regions were affected less drastically, possibly because they are adapted to the environmental stresses characteristic of their habitats. Our results did not support these patterns.

During drought, we observed lower richness of resident species at both of our study sites. There are several possible explanations for this. It is possible that higher temperatures and less precipitation during drought 
lead to a reduction in available resources, making productivity and survival more difficult (Bolger et al. 2005). Birds may also move to other parts of the landscape where water is artificially supplemented, although this may not be likely in the region we studied, as urbanized habitat is a barrier to dispersal of many resident birds of coastal sage scrub (Bolger et al. 1991). For species that are more dispersive or migratory, however, some examples of movement in response to drought are known in southern California. The two largest invasions of the White-breasted Nuthatch (Sitta carolinensis) in San Diego County outside of a its breeding range occurred in 1961 and 2002, after the two of the driest years in that county's history (Unitt 2004). Migrating Lazuli Buntings (Passerina amoena), normally scarce in urban areas of coastal southern California, were common in the city of San Diego in spring 2006, after the very dry winter of 2005-06 (P. Unitt pers. comm.)

In the absence of suitable refugia, resident species may succumb because of increased mortality and/or reduced reproductive output over several years (Craig and Chapman 2003). During extended droughts, clutches of birds with access to supplemental water may not decline as steeply (Hudgens et al. 2009). Even at the Bernard Field Station, however, with its supplemental water source, the number of resident species declined somewhat during the drought, although the decline was greater at the Voorhis Reserve, which lacks supplemental water. Thus, the presence of supplemental water may help alleviate drought stress in resident birds (Crooks et al. 2004). Reductions in reproductive output and increased mortality during drought may also be due to a reduced food supply for birds (Bolger et al. 2005).

Reduced detectability is another possible explanation for the reduction in number of resident species during drought: as birds reduce reproductive behavior, they may become more cryptic and difficult to detect (Bolger et al. 2005). With the data available, we are not able to determine whether declines in species richness are due to decreased detectability, decreased abundance, or true presence or absence. Investigation of the exact mechanisms causing such reductions remains a subject for future study.

Decreased reproductive output during a drought presents another factor affecting population persistence. Extended droughts that depress reproductive output in several consecutive years may lead to population declines and push small isolated populations over the brink of extirpation. For example, coastal southern California was in either a "severe" or "extreme" drought from June 2012 to January 2017 (Palmer 1965, NOAA 2017). Drought and heat stress intensified by climate change contribute to the decline and extirpation of species (Cahill et al. 2012), as exemplified by a 16-year study of the Burrowing Owl (Athene cunicularia) near Albuquerque, New Mexico, which found a correlation between drought and delayed breeding, decreased mass of both adults and young, and a 98\% decline of the population (CruzMcDonnell and Wolf 2015).

The number of species of migrants at the Voorhis Reserve in drought and nondrought periods did not differ significantly, and at the Bernard Field Station the richness of migrant species increased during drought, possibly because of the availability of supplemental water. Regardless of the presence of drought, species richness of migrants was much higher at the Bernard Field Station (7.7 species per survey) than at the Voorhis Reserve (1.6 
species per survey). We suggest these results could illustrate an influence of supplemental water, which may provide migrants refugia from drought.

The effects of climate change on southern California landscapes are leading to profound changes in native communities. Intensified drought means that resident bird species will need to either acclimate to these conditions or disperse to areas with more favorable habitat. Restoration and management, such as providing supplemental water, may be an important consideration for maintaining the diversity of bird species in some regions. Our study provides analyses of long-term data that demonstrate how bird communities respond to a changing environment over time.

\section{LITERATURE CITED}

Albright, T. P., Pidgeon, A. M., Rittenhouse, C. D., Clayton, M. K., Flather, C. H., Culbert, P. D., Wardlow, B. D., and Radeloff, V. C. 2010. Effects of drought on avian community structure. Global Change Biol. 16:2158-2170; doi 10.1111/j.1365-2486.2009.02120.x.

Allen, L. W., Garrett, K. L., and Wimer, M. C. 1994. Los Angeles County Breeding Bird Atlas. Los Angeles Audubon Soc., Los Angeles.

Atwood, J. L. 1993. California Gnatcatchers and coastal sage scrub: The biological basis for endangered species listing, in Interface between Ecology and Land Development in California (J. E. Keeley, ed.), pp. 149-169. S. Calif. Acad. Sci., Los Angeles.

Barr, K. R., Kus, B. E., Preston, K. L., Howell, S., Perkins, E., and Vandergast, A. G. 2015. Habitat fragmentation in coastal southern California disrupts genetic connectivity in the Cactus Wren (Campylorhynchus brunneicapillus). Molec. Ecol. 24:2349-2363; doi 10.1111/mec.13176.

Bennett, J. M., Nimmo, D. G., Clarke, R. H., Thomson, J. R., Cheers, G., Horrocks, G. F., Hall, M., Radford, J. Q., Bennet, A. F., and MacNally, R. 2014. Resistance and resilience: Can the abrupt end of extreme drought reverse avifaunal collapse? Diversity and Distributions 20:1321-1332; doi 10.1111/ddi.12230.

Berthold, P. 2001. Bird Migration: A General Survey. Oxford Univ. Press, Oxford, England.

Bock, C. E., and Bock, J. H. 1999. Response of winter birds to drought and shortduration grazing in southeastern Arizona. Cons. Biol. 13:1117-1123; doi 10.1046/j.1523-1739.1999.98313.x.

Bolger, D. T., Alberts, A. C., and Soulé, M. E. 1991. Occurrence patterns of bird species in habitat fragments: Sampling, extinction, and nested species subsets. Am. Nat. 137:155-166.

Bolger, D. T., Patten, M. A., and Bostock, D. C. 2005. Avian reproductive failure in response to an extreme climatic event. Oecologica 142:398-406; doi 10.1007/ s00442-004-1734-9.

Cahill, A. E., Aiello-Lammens, M. E., Fisher-Reid, M. C., Hua, X., Karanewsky, C. J., Ryu, H. Y., Sbeglia, G. C., Spagnolo, F., Waldron, J. B., Warsi, O., and Wiens, J. J. 2012. How does climate change cause extinction? Proc. Royal Soc. B 280:20121890: doi 10.1098/rspb.2012.1890.

Chase, M. K., Kristan, W. B. III, Lynam, A. J., Price, M. V., and Rotenberry, J. T. 2000. Single species as indicators of species richness and composition in California coastal sage scrub birds and small mammals. Cons. Biol. 14:474-487; doi 10.1046/j.1523-1739.2000.98312.x.

Craig, M. D., and Chapman, A. 2003. Effects of short-term drought on the avifauna of Wanjarri Nature Reserve: What do they tell us about drought refugia? J. Royal Soc. W. Australia 86:133-137. 
Crooks, K. R., Suarez, A. V., and Bolger, D. T. 2004. Avian assemblages along a gradient of urbanization in a highly fragmented landscape. Biol. Cons. 115:451-462; doi 10.1016/S0006-3207(03)00162-9.

Cruz-McDonnell, K. K., and Wolf, B. O. 2015. Rapid warming and drought negatively impact population size and reproductive dynamics of an avian predator in the arid southwest. Global Change Biol. 22:237-253; doi 10.1111/gcb.13092.

Faaborg, J. 1982. Avian population fluctuations during drought conditions in Puerto Rico. Wilson Bull. 94: 20-30.

George, T. L., Fowler, A. C., Knight, R. L., and McEwen, L. C. 1992. Impacts of a severe drought in grassland birds in western North Dakota. Ecol. Appl. 2:275-284; doi 10.2307/1941861.

Goldstein, L. J., and Suding, K. N. 2014. Intra-annual rainfall regime shifts competitive interactions between coastal sage scrub and invasive grasses. Ecology 95:425435; doi 10.1890/12-0651.1.

Griffin, D., and Anchukaitis, K. J. 2014. How unusual is the 2012-2014 California drought? Geophys. Res. Lett. 41:9017-90231; doi 10.1002/2014GL062433.

Herremans, M. 2004. Effects of drought on birds in the Kalahari, Botswana. Ostrich 75:217-227; doi 10.2989/00306520409485448.

Hudgens, B. R., Johnston, N. N., Bradley, J. E., and Bridges, A. S. 2009. Benefits of supplemental feeding are climate dependent in the San Clemente Loggerhead Shrike, in Proceedings of the 7th California Islands Symposium (C. C. Damiani and D. K. Garcelon, eds.), pp. 315-325. Inst. Wildlife Studies, Arcata, CA.

Mastrandrea, M. D., and Luers, A. L. 2012. Climate change in California: Scenarios and approaches for adaptation. Climatic Change 111:5-16; doi 10.1007/ s10584-011-0240-4.

McCaull, J. 1994. The Natural Community Conservation Planning Program and the Coastal Sage Scrub Ecosystem of Southern California: Environmental Policy and Biodiversity. Island Press, Washington, D.C.

McCreedy, C., van Riper, C., III, Esque, T. C., and Darrah, A. J. 2015. Effects of drought and fire on bird communities in the Kofa National Wildlife Refuge, Arizona. U.S. Geol. Surv. Open-File Rep. 2015-1240; doi 10.3133/ofr20151240.

Minnich, R. A., and Dezzani, R. J. 1998. Historical decline of coastal sage scrub in the Riverside-Perris Plain, California. W. Birds 29:366-391.

Moriarty, D. J. 2013. A twenty-year investigation of the effects of fire on a coastal sage scrub bird community. W. Birds 44:2-16.

Moriarty, D. J., Farris, R. E., Noda, D. K., and Stanton, P. A. 1985. Effects of fire on a coastal sage scrub bird community. Southwest. Nat. 30:452-453; doi $10.2307 / 3671284$.

National Oceanic and Atmospheric Administration (NOAA). 2017. Historical Palmer drought indices. U.S. Dept. of Commerce; www.ncdc.noaa.gov/temp-andprecip/drought/docs/palmer.pdf.

National Wildlife Federation. 2008. Increased risk of catastrophic wildfires: Global warming's wake-up call for the western United States. Natl. Wildlife Fed., Reston, VA; www.nwf.org/ /media/PDFs/Global-Warming/NWF_WildFiresFinal.ashx.

Palmer, W. 1965. Meteorological drought. U.S. Weather Bureau Res. Pap. 45; www.ncdc.noaa.gov/temp-and-precip/drought/historical-palmers.php.

Patten, M. A., and Rotenberry, J. T. 1999. The proximate effects of rainfall on clutch size of the California Gnatcatcher. Condor 101:876-880; doi $10.2307 / 1370080$.

R Core Team. 2015. R: A language and environment for statistical computing. R Foundation for Statistical Computing, Vienna, Austria; www.R-project.org.

Robeson, S. M. 2015. Revisiting the recent California drought as an extreme value. Geophys. Res. Lett. 42:6771-6779; doi 10.1002/2015GL064593.

Rubinoff, D. 2001. Evaluating the California Gnatcatcher as an umbrella species for 
conservation of southern California coastal sage scrub. Cons. Biol. 15:13741383; doi 10.1046/j.1523-1739.2001.00176.x.

Sedgwick, J. A. 2004. Site fidelity, territory fidelity, and natal philopatry in Willow Flycatchers (Empidonax traillii). Auk 121:1103-1121; doi 10.1642/0004-8038(2004)121[1103:SFTFAN]2.0.CO;2.

Stracey, C. M. 2010. Pattern and process in urban bird communities: What makes the Northern Mockingbird an urban adapter? Ph.D. dissertation, Univ. of Fla., Gainesville.

Taylor, R. S. 2005. A new look at coastal sage scrub: What 70-year-old VTM plot data tells us about southern California shrublands, in Planning for biodiversity: Bringing research and management together (B. E. Kus and J. L. Beyers, tech. coords.), pp. 55-77. US Forest Service Gen. Tech. Rep. PSW-GTR-195, Pac. Southwest Res. Sta., Albany, CA; http://citeseerx.ist.psu.edu/viewdoc/down load?doi=10.1.1.224.1156\&rep=rep1\&type $=$ pdf\#page $=67$.

U.S. Geological Survey. 2005. The National Atlas of the United States of America. California; http://maps.redcross.org/website/Maps/Images/California/ pageprecip_ca3.pdf.

Unitt, P. 2004. San Diego County bird atlas. Proc. San Diego Soc. Nat. Hist. 39.

Wang, S. Y., Hipps, L., Gillies, R. R., and Yoon, J. H. 2014. Probable causes of the abnormal ridge accompanying the 2013-2014 California drought: ENSO precursor and anthropogenic warming footprint. Geophys. Res. Lett. 41:32203226; doi 10.1002/2014GL059748.

Winter, K., and Hargrove, L. 2004. Gray Vireo (Vireo vicinior), in The coastal scrub and chaparral bird conservation plan: A strategy for protecting and managing coastal sage scrub and chaparral habitats and associated birds in California. California Partners in Flight; www.prbo.org/calpif/htmldocs/species/scrub/gray_vireo.htm.

Accepted 14 March 2019

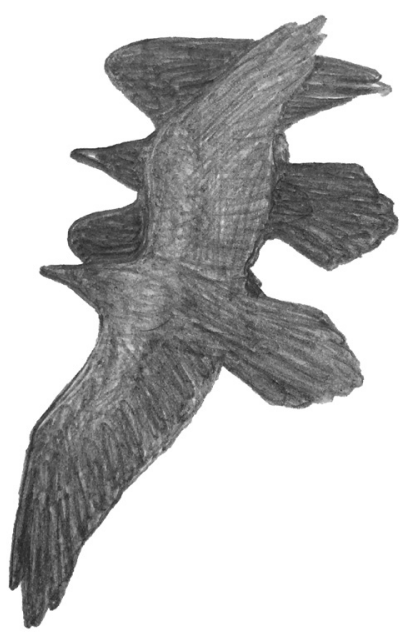

Common Ravens

Sketch by Teodelina Martelli 